\title{
EDITORIAL NOTE Swelling and Shrinking of Porous Materials: From Colloid Science to Poromechanics
}

\author{
MARCIO A. MURAD \\ Laboratório Nacional de Computação Científica LNCC/MCT - Petrópolis, RJ, Brasil
}

Electrochemical interaction between colloidal particles and an aqueous solution is a central subject in Colloid Science. Such interactions give rise to electrokinetic phenomena in electrically charged porous media, and have received considerable attention with a wide range of applications in different fields of science and engineering. Several porous materials composed of electrically charged macromolecules saturated by saline solutions may undergo swelling by fresh water uptake. Among this class of colloidal systems, we highlight 2-1 lattice clays, hydrophilic polymers, gels, shales, corneal endothelium and connective biological tissues. For example, the response of smectite 2:1 clay particles to changes in water content has been studied for decades. Such phenomenon plays a critical role in the quality of groundwater, in the effectiveness of some clean up technologies and in the distribution of plants and nutrients in the Earth's crust. Clay swelling (or collapse) is of widespread relevance in geotechnical and geoenvironmental fields. Upon inundation, it may have undesired consequences, as they heave upward upon hydration (or shrink upon desiccation) causing damage to the foundations of buildings ranging from minor cracking to irreversible displacements of footings. Moreover, swelling and deterioration of shales are the major contributors to drilling problems and are key factors in the wellbore stability management. Finally, due to their low hydraulic conductivity, plasticity, swelling and adsorptive capacity for contaminants, bentonitic based compacted clays have been used as sealing materials to inhibit the migration of contaminants to the environment, and have been also considered to investigate the disposal of high-level radioactive waste in various countries.

Beyond applications in natural geomaterials, swelling polymers/biopolymers have numerous technological applications, such as size exclusion chromatography, gel electrophoresis in filtration processes, development of efficient drug delivery substrates, in contact lenses, in semiconductor manufacturing and in food stuffs. In biomedical technology, electrical interactions between ions and negatively charged proteoglycans rule the deformation of cartilaginous soft hydrated tissues as load-bearing structures. The swelling property is tied-up to the physiological states of soft connective tissues (articular cartilage and intervertebral disk) and plays an important role in articulating joint lubrication and damping of dynamic forces in the human body.

The fundamental thermodynamic processes underlying electrokinetic phenomena involve several couplings, such as: transport of mobile ions near charged surfaces (electro-migration), flow and motion of charged particles driven by an electric field (electro-osmosis and electrophoresis), dissolution/precipitation reactions, electrolysis of water, sorption, desorption and protonation/deprotonation chemical reactions. Owing to their complexity, it is imperative that any macroscopic model describing these electro-chemo-mechanical interactions inherent to this type of system contains accurate constitutive relations.

During the past few decades, a significant amount of research has been developed towards the derivation of models capable of capturing coupled electro-chemo-hydro-mechanical effects in charged porous media. Owing to the aforementioned complex fine-scale electro-chemical interactions, the accuracy of purely macroscopic models 
may deteriorate. In order to better understand the correlation between local electro-chemistry and the macroscopic response of the medium, it becomes essential to develop multiscale models. Multiscale methods are capable of establishing accurate correlations among properties at different scales, and allow to bridge the fields of macroscopic Soil Mechanics and nanoscopic Colloid Science.

The content of the articles within this issue illustrates the applications of colloidal systems at different length scales, and emphasizes the necessity of developing multiscale methods for further developing of appropriate constitutive laws. The selected papers published herein were presented at the IUTAM Symposium on "Swelling and Shrinking of Porous Materials: From Colloid Science to Poromechanics", which took place at the National Laboratory for Scientific Computing of the Ministry of Science and Technology (LNCC/MCT) in Petrópolis, Brazil, during the period of August 06-10, 2007.

The realization of this symposium after its approval by the IUTAM general assembly was a great accomplishment for the Brazilian scientific community, as it recognized the ongoing efforts towards the nucleation of a multidisciplinary forum capable of providing promising discussions among the participants on the intriguing and challenging problems that commonly arise in the field of colloidal porous materials.

In this issue, the reader will find applications of electrically charged porous media at a wide range of scales from Angstroms (Nanoscale) to Kilometers (Field Scale). Articles included in Chemical and Biological Sciences (see e.g. Dufreche et al. 2010, Lima et al. 2010 ) treat the problem at the nanoscopic scale, where physico-chemical and electro-chemical effects naturally appear, giving rise to up-scaled anomalous phenomena. Some of the papers published in Physical Sciences concentrate their efforts on the mesoscale computational modeling of expansive porous media, such as clays and gels (see e.g. Narsilio et al. 2010). The application in Medical Sciences focuses on biological tissues, such as intervertebral disc and osteo-articular porous materials at coarser scale where the fluid in the nano-pores and the solid matrix have been homogenized to give rise to a mesoscopic description (Lemaire et al. 2010). Finally, the articles in Engineering and Earth Sciences deal with applications in soil and rock mechanics, and are mainly related to contaminant transport in clays (Sanchez et al. 2010) and wellbore stability in shales (Nguyen and Abousleiman 2010). Such applications in geological formations occur at much coarser scales, and their effective behavior exhibits strong correlations with the aforementioned nano-scale electro-chemical phenomena.

To summarize, the challenge here is bridging these phenomena at different scales in order to enhance our knowledge on colloidal systems and, consequently, predict their behavior computationally. Whence, I have great expectations that the refereed papers published herein will be the basis for future work on this genuine multidisciplinary area.

\section{REFERENCES}

Dufrêche J-F, Rotenberg B, MARry V And Turq P. 2010. Bridging molecular and continuous descriptions: the case of dynamics in clays. An Acad Bras Cienc 82: 61-68.

Lemaire T, NAili S And SAnSAlone V. 2010. Multiphysical modelling of fluid transport through osteo-articular media. An Acad Bras Cienc 82: 127-144.

Lima ERA, Biscaia JR. EC, Boström M AND TAVARES FW. 2010. Ion-specific thermodynamical properties of aqueous proteins. An Acad Bras Cienc 82: 109-126.

NARsilio GA, Smith DW And Pivonka P. 2010. Estimating vertical and lateral pressures in periodically structured montmorillonite clay particles. An Acad Bras Cienc 82: 13-24.

NGUYEN VX AND ABOUSLEIMAN YN. 2010. Incorporating electrokinetic effects in the porochemoelastic inclined wellbore formulation and solution. An Acad Bras Cienc 82: 195-222.

SÁnchez M, Gens A And Olivella S. 2010. Effect of thermo-coupled processes on the behaviour of a clay barrier submitted to heating and hydration. An Acad Bras Cienc 82: 153-168. 